Journal of Computer Science 8 (4): 551-555, 2012

ISSN 1549-3636

(C) 2012 Science Publications

\title{
Temperature Effect on Uncooled Semiconductor Laser Diode to the Network Performance System
}

\author{
Mohammad Syuhaimi Ab-Rahman and Nurain Izzati Shuhaimi \\ Department of Electrical, Electronics and Systems, \\ Engineering and Built Environment, \\ University Kebangsaan Malaysia, 43600 UKM Bangi, Selangor, Malaysia
}

\begin{abstract}
Problem statement: The characteristics of a laser diode are highly dependent on the temperature of the laser chip. Thus, the effect of temperature on the network performance of uncooled semiconductor laser diode are studied by simulating its equivalent electrical circuit, developed from the rate equations that governing optical components directly into an electrical simulation framework. Approach: The simulations are carried out using circuit analysis program named OptiSPICE and OptiSystem. In this simulation study, the analysis is done based on four parameters in order to determine the quality of received signal which are; bit error rate, eye height, maximum $\mathrm{Q}$ factor and jitter. Results: As the temperature variance is fed to the laser diode through an external thermal noise, the result will shows that the ambient temperature of laser diode is varied with time. In this case, the performance of laser diode will decreases as the operating temperature increases or, conversely the performance increases as the operating temperature decreases. The circuit design must be simulated using a SPICE engine that incorporates both electrical and optical models, which is OptiSPICE software. It cannot be simulated using traditional software packages because they do not provide sufficient coupling between electrical and optical devices. Conclusion: We successfully analyze the effect of the temperature on laser source to the performance of optical network system.
\end{abstract}

Key words: Semiconductor laser diode, temperature effect, bit error rate, eye height, maximum Q factor, controlling jitter, quantum mechanics, laser beam, laser diodes, gas lasers, mirror alignment, photosensitive sensitivity

\section{INTRODUCTION}

The term laser is an acronym for Light Amplification by the Stimulated Emission of Radiation; device which is built on the principles of quantum mechanics to create a beam of light where all of the photons are in a coherent state, usually with the same frequency and phase Principal of Laser, 2010. Many lasers deliver light in an almost-perfectly parallel beam (collimated) that is very pure, approaching a single wavelength. In addition, a laser is capable of producing an intense beam of photons having identical scalar and vector properties (frequency, phase, direction and polarization). As a result, a laser beam can be bright, monochromatic, coherent and unidirectional (Malacara and Thompson, 2001).

The commercial and industrial use of laser diodes has dramatically increased recently. The optical characteristics, small size and ruggedness of laser diodes have allowed many new uses to be commercialized. The output of laser diodes is very bright considering their small size. Today, hundreds of watts of power are commercially available from laser diodes operating under continuous wave conditions in packages as small as a few cubic inches. This characteristic makes these devices suitable for cable TV transmission, High Definition TV (HDTV) development and medical applications. Since laser diodes are made of semiconductor materials, they do not require the fragile glass enclosures or mirror alignment typical of gas lasers. The resulting ruggedness and small size allow laser diodes to be used in environments and spaces in which other types of lasers cannot operate. In addition, laser diode also can be directly modulated at high frequencies. By modulating the drive current, the output of the laser diode is modulated with frequencies up to several $\mathrm{GHz}$ in high-speed data communications Laser Diode Technology, 2011.

In this simulation study, focus is on Semiconductor Laser Diode (SLD). SLDs lasing at 1.3 and $1.55 \mathrm{~lm}$ -

Corresponding Author: Mohammad Syuhaimi Ab-Rahman, Department of Electrical, Electronics and Systems, Engineering and Built Environment, University Kebangsaan Malaysia 43600 UKM Bangi, Selangor, Malaysia 
band are mainly used as the optical sources and their reliability are very important for maintaining their performance in the systems (Ab-Rahman and Hassan, 2010a). The improvements in laser manufacture today allow operating uncooled direct modulated SLDs over abroad temperature range (Ab-Rahman and Hassan, 2010b). Uncooled SLD are simply wide temperature of operation range, where it is designed to be wider than the span of the environmental operating conditions of communications equipment in which they are used. Uncooled SLD enable low-cost packaging by eliminating many items such as cooler, the thermistor which indicates SLD temperature at any time and the thermal control loop (Ab-Rahman and Hassan, 2010c). Because a strong cost reduction in optical systems is achieved when eliminating costly laser temperature control, the uncooled SLDs have been considered as a key technology for future optical network systems (AbRahman and Hassan, 2011). When considering of uncooled SLD in high speed optical communication system, it is high of importance to keep the temperature variation as optimum as possible because increasing temperature will introduces decreasing of the features of SLD.

The heart of novelty of the study is that the introduced simulations provide a helpful basis for interpreting the experimental results. This study also demonstrated detailed profiles of optical data pattern and eye diagram in directly modulated laser diodes. This simulation study is then focused on the eye diagram parameter analysis in the laser performance during the working condition. The eye diagram shown in Fig. 1 is used to analyze the working condition in the laser network. The analysis is done based on four parameters in order to determine the quality of received signal. The parameters are bit error rate, eye height and maximum Q factor and jitter (Ab-Rahman et al., 2011). Eye height: It refers to the distance from the base to the peak of the eye measured in voltage. The allowed minimum height value of the eye diagram is inversely proportional to the photosensitive sensitivity. High photosensitive sensitivity could assess data at low height value of eye diagram whereas low photosensitive sensitivity requires high eye diagram height value for data assessment (for example, at photosensitive sensitivity-22.8 dBm, the eye diagram height value is $5.5 \mu \mathrm{V}$ and at sensitivity- $18 \mathrm{dBm}$, the allowable eye diagram height value is $10 \mu \mathrm{V}$ ). If there is any fixation on the photosensitive sensitivity value, the increase in data transmission rate will give similar eye diagram height value.

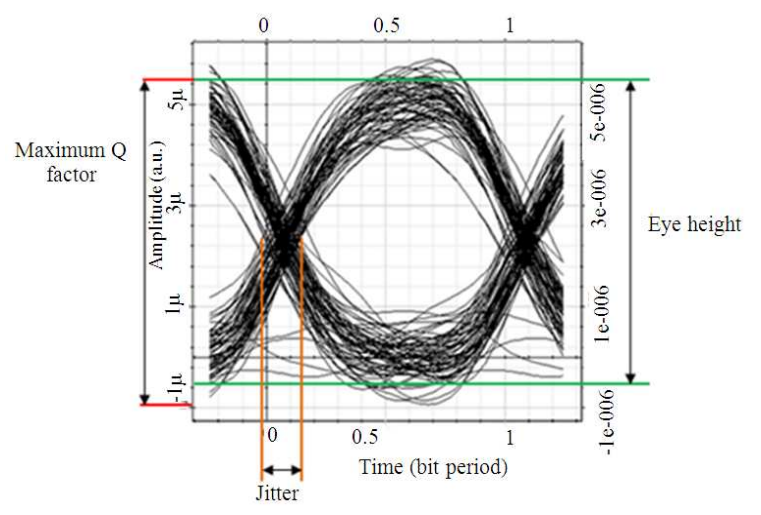

Fig. 1: Eye diagram parameters which determine the quality of receive signal

Maximum Q factor: The maximum $Q$ factor refers to the quality of the produced eye diagram to be analyzed. This value is fixed and equal for all photosensitivity values for various data transmission rate. The allowable maximum $\mathrm{Q}$ factor is 6 in current communication system to obtain BER value equivalent to $1 \times 10^{-9}$.

Jitter: Deterministic jitter refers to the shifting which occurs on derivative and embedded time of the received signal and the value is measured according to the duration at the crossing point. It is measured in UI (unit interval) unit and the allowable maximum value is 0.2 UI. Normally the jitters are generated and give pronounce impact on the data transmission system of more than 1 Gbps.

Eye area: Eye area refers to the distance between levels of bit 0 and bit 1 and the distance between right and left embedded derivatives time crossing. The parameters are applied in mask technique to evaluate the quality of the received signal. The width of this area is important in the process of differentiating bit 1 and bit 0 as well as the sequence of the first and second bits. The more wide the eye an area is, the bigger the received data quality is and thus, the process of signal sampling becomes easier.

\section{MATERIALS AND METHODS}

The main interface is the OptiSpice schematic, where it is one of among Opt wave design software for photonic. Opti SPICE is circuit design software for analysis of integrated circuits including interactions of optical and electronic components and it is a fullyintegrated solution for parameter extraction, schematic capture, circuit simulation and waveform analysis Optiwave System Inc, 2010. 
Table 1: Summary of temperature description by thermal source

\begin{aligned} \hline DC value (C) & Time-temperature values (s, C) \\ \hline 0 & $\{00\}\{1 \mathrm{e}-60\} \\ 20 & \{00\}\{1 \mathrm{e}-60\}\{2 \mathrm{e}-620\}\{4.5 \mathrm{e}-620\} \\ 50 & \{00\}\{1 \mathrm{e}-60\}\{2 \mathrm{e}-620\}\{4.5 \mathrm{e}-620\} \\ 70 & \{5.5 \mathrm{e}-650\}\{8 \mathrm{e}-650\} \\ & \{00\}\{1 \mathrm{e}-60\}\{2 \mathrm{e}-620\}\{4.5 \mathrm{e}-620\} \\ & \{5.5 \mathrm{e}-650\}\{8 \mathrm{e}-650\}\{9 \mathrm{e}-670\}\{11.5 \mathrm{e}-670\} \\ & \{00\}\{1 \mathrm{e}-60\}\{2 \mathrm{e}-620\}\{4.5 \mathrm{e}-620\}\{5.5 \mathrm{e}-650\} \\ & \{8 \mathrm{e}-650\}\{9 \mathrm{e}-670\}\{11.5 \mathrm{e}-670\} \\ & \{12.5 \mathrm{e}-6100\}\{15 \mathrm{e}-6100\} \\ & \{00\}\{1 \mathrm{e}-60\}\{2 \mathrm{e}-620\}\{4.5 \mathrm{e}-620\} \\ & \{5.5 \mathrm{e}-650\}\{8 \mathrm{e}-650\}\{9 \mathrm{e}-670\}\{11.5 \mathrm{e}-670\} \\ & \{12.5 \mathrm{e}-6100\}\{15 \mathrm{e}-6100\} \\ & \{16 \mathrm{e}-6120\}\{18.5 \mathrm{e}-6120\} \\ & \{5.5 \mathrm{e}-650\}\{8 \mathrm{e}-60\}-650\}\{9 \mathrm{e}-670\}\{11.5 \mathrm{e}-670\} \\ & \{12.5 \mathrm{e}-6100\}\{15 \mathrm{e}-6100\}\{16 \mathrm{e}-6120\} \\ & \{18.5 \mathrm{e}-6120\}\{19.5 \mathrm{e}-6150\}\{22 \mathrm{e}-6150\} \\ & \{00\}\{1 \mathrm{e}-60\}\{2 \mathrm{e}-620\}\{4.5 \mathrm{e}-620\} \\ & \{5.5 \mathrm{e}-650\}\{8 \mathrm{e}-650\}\{9 \mathrm{e}-670\}\{11.5 \mathrm{e}-670\} \\ & \{12.5 \mathrm{e}-6100\}\{15 \mathrm{e}-6100\}\{16 \mathrm{e}-6120\} \\ & \{18.5 \mathrm{e}-6120\}\{19.5 \mathrm{e}-6150\}\{22 \mathrm{e}-6150\} \\ & \{23 \mathrm{e}-6170\}\{25.5 \mathrm{e}-6170\} \\ & \{00\}\{1 \mathrm{e}-60\}\{2 \mathrm{e}-620\}\{4.5 \mathrm{e}-620\} \\ & \{5.5 \mathrm{e}-650\}\{8 \mathrm{e}-650\}\{9 \mathrm{e}-670\} \\ & \{11.5 \mathrm{e}-670\}\{12.5 \mathrm{e}-6100\}\{15 \mathrm{e}-6100\} \\ & \{16 \mathrm{e}-6120\}\{18.5 \mathrm{e}-6120\}\{19.5 \mathrm{e}-6150\} \\ & \{22 \mathrm{e}-6150\}\{23 \mathrm{e}-6170\}\{25.5 \mathrm{e}-6170\} \\ & \{26.5 \mathrm{e}-6200\}\{28 \mathrm{e}-6200\} \\$\hline & \end{aligned}

Table 2: Layout parameter's detail in OptiSystem GUI

\begin{tabular}{ll}
\hline Parameter & Value \\
\hline Bit rate & $1 \mathrm{e} 9 \mathrm{bits} / \mathrm{sec}$ \\
Sequence length & $128 \mathrm{bits}$ \\
& \\
Samples per bit & 64 \\
No. of samples & 8192 \\
\hline
\end{tabular}

The process to design a circuit of uncooled SLD by presence of temperature variation is start by using OptiSPICE schematic framework, where it is illustrated in Fig. 2. The circuit design in OptiSPICE schematic is then visualized in OptiSystem Graphical User Interface (GUI) to get the simulation results, as shown in Fig. 3.

In OptiSPICE schematic framework design, the initial DC current is kept at 0.078A and only the wavelength is changed, where three different wavelengths have been used to analyse the laser performance, i.e., 1550,1490 and $1450 \mu \mathrm{m}$. In this design, ambient temperature of the laser is varied with time as shown in Fig. 4. This temperature variance is fed to the laser through an external thermal source and for each wavelength; the summary of injected temperature is provided in Table 1. While in OptiSystem GUI, the attenuation at optical attenuator is maintained at $28.5 \mathrm{~dB}$ and layout parameters are changed, as listed in Table 2.

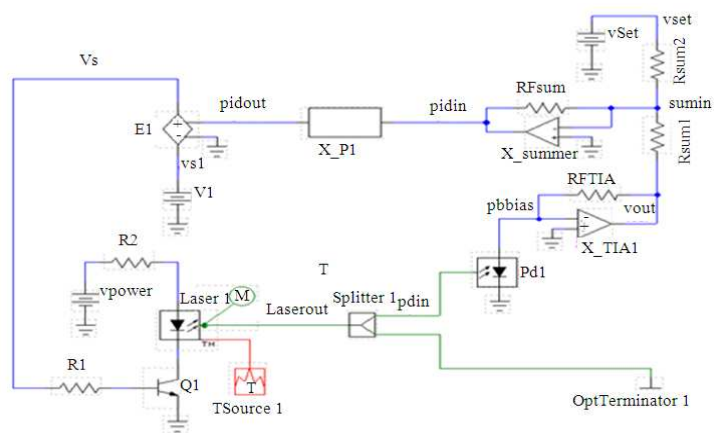

Fig. 2: Point-to-point configuration of SLD design

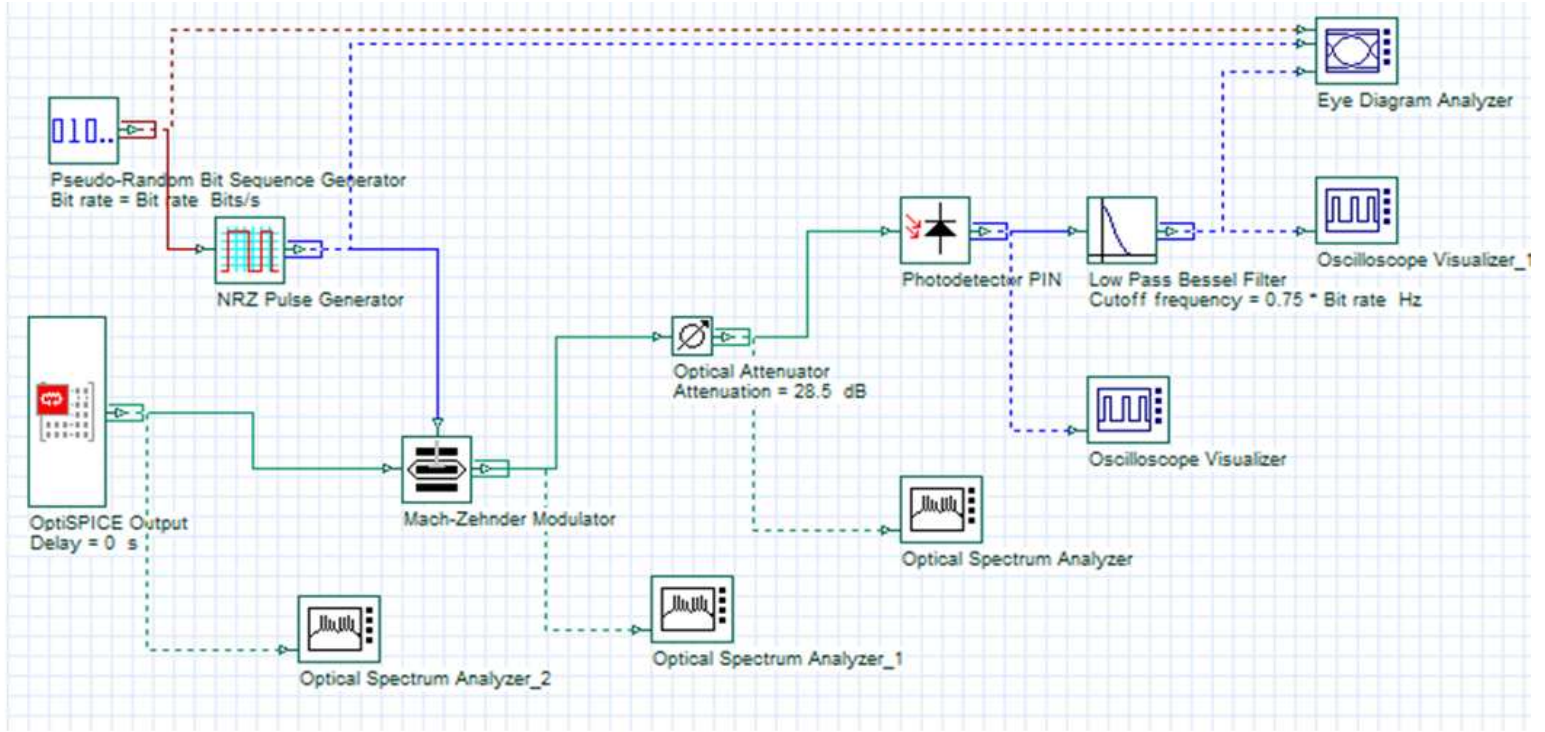

Fig. 3: Connection of components 


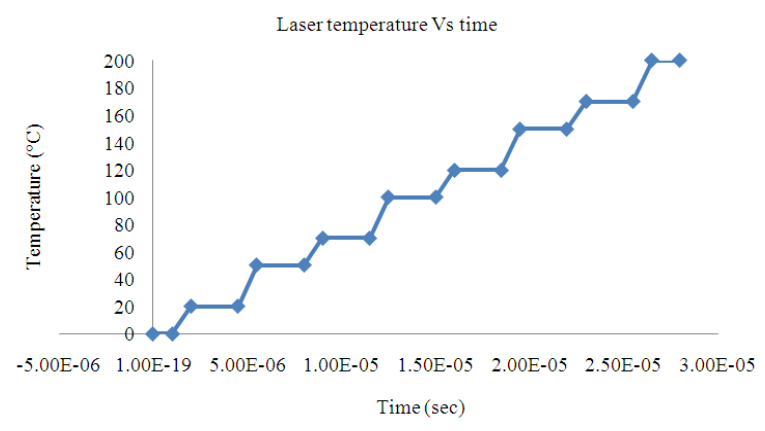

Fig. 4: Laser temperature

Once the setting of layout is done, then the simulation result of three parameters which are $\mathrm{Q}$ factor, minimum bit error rate, eye height and jitter; is carried out by clicking at Eye Diagram Analyzer in OptiSystem GUI. Note that, eye diagram is qualitative measure of system performance.

\section{RESULTS}

Figure 5-8 show the simulated result of point-topoint configuration of SLD design for three different wavelengths; 1550, 1490 and $1450 \mathrm{~nm}$, which respectively for $\mathrm{Q}$ factor, minimum bit error rate, eye height and jitter.

\section{DISCUSSION}

By looking at Fig. 5, it shows that the Q factor of a laser diode is inversely proportional to its operating temperature.

Theoretically, the Q-factor is a parameter that directly reflects the quality of a digital optical communications signal. The lower the Q-factor, the worst the quality of an optical signal. Thus, it proves that as temperature increases, the $\mathrm{Q}$ factor of the laser diode going decreases. Higher $Q$ factor indicates a lower rate of energy loss relative to the stored energy of the laser diode, where high $\mathrm{Q}$ factor value means that the energy is stored well inside the cavity while, low Q factor value means that the energy is emitted from the cavity rapidly Eningen GmbH, 2011.

For this reason, maintaining the higher $\mathrm{Q}$ factor of operation is recommended. If not, the temperature of operation will affect seriously the characteristics of SLD.

In the other case, for every different wavelength, the minimum BER value is changed relatively to the different value of operating temperature. Theoretically, BER is a ratio of number of bit received in error to the total number of bits transmitted.

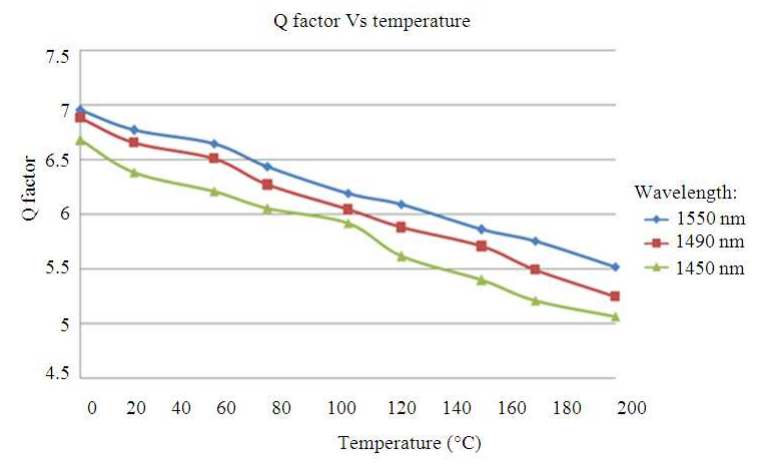

Fig. 5: Effects of temperature on $Q$ factor

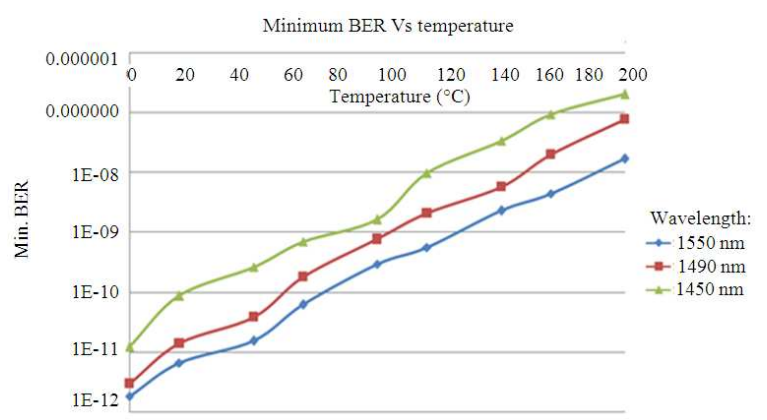

Fig. 6: Effects of temperature on minimum

From the result in Fig. 6, it shows that the higher the operating temperature of laser diode, the higher the minimum BER will be. Note that, the difference in the mean values produces the vertical eye opening. The higher the difference, the better the BER will be as the two bell curves drift away from each other and have less overlap. This difference is divided by the sum of the noise distributions which are represented by the width of the bell curves. Increases in noise result in more overlap in the two bell curves resulting in a higher BER. Typically, the bit error rate is strongly dependent on the transmitted power and the latter must be high enough to keep the bit error rate below a certain acceptable level; e.g., $10^{-12}$ for earth-based telecommunication systems or $10^{-6}$ for satellite control (Paschotta, 2008).

The variation of operating temperature also affected on the eye height of laser diode. Figure 7 shows how the eye height curve changes with operating temperature for typical laser diodes, where the relationship for the laser diode tested in this simulation is plotted; the increase in eye height corresponds to different wavelengths with increasing operating temperature. As mentioned earlier, eye height is a measure of the vertical opening of an eye diagram. 


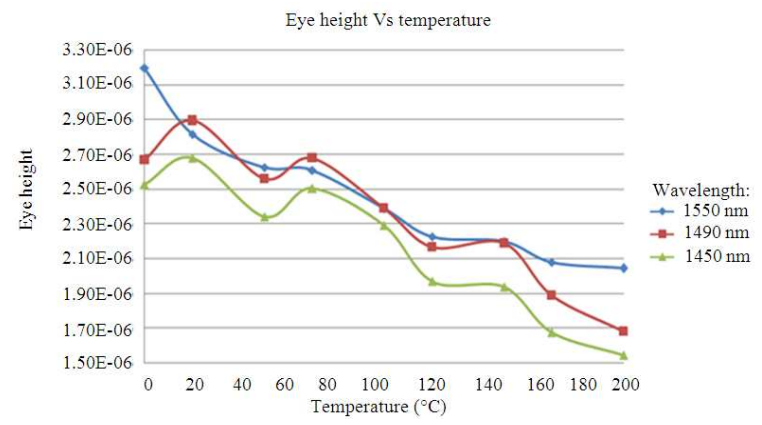

Fig. 7: Effects of temperature on eye height

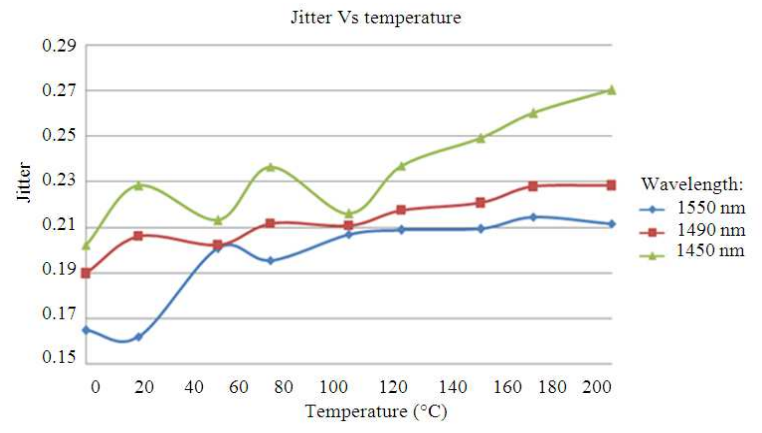

Fig. 8: Effects of temperature on jitter

An ideal eye opening would be measured from the one level to the zero level. However, noise on the eye will cause the eye to close. The eye height measurement determines eye closure due to noise. The better quality the digital signal transmission, the more open eye there should be in the eye. Said differently, the eye width and eye height should be as large as possible to obtain the most efficient performance of laser diode.

Jitter is one of key performance factor in highspeed data communications such as in laser diode application. It is a must to maintain a jitter in order to minimize the probability of incurring a BER, as mentioned before, defined as the ratio of bad bits incurred to the number of valid bits in the data pattern. Controlling jitter in the digital signal path plays a very important role in minimizing BER and thereby increasing system reliability. As a result, it is important to understand jitter, its sources and its effects on a system. Said differently; as the demand for higher frequencies and data rates increase, so does the demand for lower jitter. Thus, by referring to Fig. 8, it proves that the jitter is getting lower as the operating temperature is lower.

\section{CONCLUSION}

In this simulation study, there was shown the important dependences of laser features on temperature. The temperature can have negative effect on the important parameters and quality of a semiconductor laser diode. There is a need to know the dependency of parameters on the temperature for successful diode construction. It is well known that a laser diode can work at a low constant temperature. In conclusion, the circuit design must be simulated using a SPICE engine that incorporates both electrical and optical models, which is OptiSPICE software. It cannot be simulated using traditional software packages that rely on cosimulation or equivalent circuit models, as they do not provide sufficient coupling between electrical and optical devices. The process is continuing until the result on effect of uncooled semiconductor laser diode in presence of temperature variation is achieved.

\section{ACKNOWLEDGEMENT}

The researchers would like to acknowledge University Kebangsaan Malaysia for supporting this research by providing equipment, material and sources of references in accomplishing this research.

\section{REFERENCES}

Ab-Rahman, M.S. and M.R. Hassan, 2010a. Theoretical analysis of the effect of temperature dependence of Auger coefficient on the turn-on time delay of uncooled semiconductor laser diodes. Optics Commun., 283: 2378-2384. DOI: 10.1016/j.optcom.2010.01.071

Ab-Rahman, M.S. and M.R. Hassan, 2010b. Temperature dependence of turn-on time delay of semiconductor laser diode: Theoretical analysis. OPTO-Elect. Rev., 18: 458-466. DOI: 10.2478/s11772-010-0015-x

Ab-Rahman, M.S. and M.R. Hassan, 2010c. Theory of cutoff temperature of operation of uncooled semiconductor laser diode. Eur. Phsical J. Applied Phys., 50: 20301-20306. DOI: 10.1051/epjap/2010034

Ab-Rahman, M.S., L. Al-Hakim Azizan, S.A.C. Aziz and K. Jumari, 2011. The eye diagram analysis of restoration scheme in FTTH-PON. J. Applied Sci., 11: 840-847.

Ab-Rahman, M.S. and M.R. Hassan, 2011. The combined effect of temperature of operation and external optical feedback on the turn-on time delay of semiconductor laser diodes. Optik-Int. J. Light Elect. Optics, 122: 266-272. DOI: 10.1016/j.ijleo.2010.01.002

Paschotta, R., 2008. Encyclopedia of Laser Physics and Technology: A-M. 1st Edn., Wiley-VCH, Weinheim, ISBN: 3527408282, pp: 434.

Malacara, D. and B.J. Thompson, 2001. Handbook of Optical Engineering. 1st Edn., Marcel Dekker, New York, ISBN: 0824799607, pp: 978. 